\title{
A Longitudinal Comparison of Arm Morbidity in Stage I-II Breast Cancer Patients Treated with Sentinel Lymph Node Biopsy, Sentinel Lymph Node Biopsy Followed by Completion Lymph Node Dissection, or Axillary Lymph Node Dissection
}

\author{
Jan J. Kootstra, MD ${ }^{1,2}$, Josette E. H. M. Hoekstra-Weebers, MSc, PhD ${ }^{3,4,5}$, Johan S. Rietman, MD, PhD ${ }^{6,7}$, Jakob de \\ Vries, MD, PhD ${ }^{1}$, Peter C. Baas, MD, PhD $^{8}$, Jan H. B. Geertzen, MD, PhD ${ }^{5,9}$, and Harald J. Hoekstra, MD, PhD ${ }^{1}$ \\ ${ }^{1}$ Department of Surgical Oncology, University Medical Center Groningen, University of Groningen, Groningen, \\ The Netherlands; ${ }^{2}$ Department of Surgery, Isala Klinieken, Zwolle, The Netherlands; ${ }^{3}$ Wenckebach Institute, University \\ Medical Center Groningen, University of Groningen, Groningen, The Netherlands; ${ }^{4}$ Comprehensive Cancer Center \\ North-East, Groningen, The Netherlands; ${ }^{5}$ SHARE, Graduate School for Health Research, University of Groningen, \\ Groningen, The Netherlands; ${ }^{6}$ Roessingh Research and Development, Enschede, The Netherlands; ${ }^{7}$ Laboratory \\ of Biomedical Engineering, Faculty of Engineering Technology, Twente University, Enschede, The Netherlands; \\ ${ }^{8}$ Department of Surgery, Martini Hospital, Groningen, The Netherlands; ${ }^{9}$ Department of Rehabilitation, University \\ Medical Centre Groningen, University of Groningen, Groningen, The Netherlands
}

\begin{abstract}
Background. Long-term shoulder and arm function following sentinel lymph node biopsy (SLNB) may surpass that following complete axillary lymph node dissection (CLND) or axillary lymph node dissection (ALND). We objectively examined the morbidity and compared outcomes after SLNB, SLNB + CLND, and ALND in stage I/ II breast cancer patients.

Materials and Methods. Breast cancer patients who had SLNB $(n=51)$, SLNB + CLND $(n=55)$, and ALND $(n=65)$ were physically examined 1 day before surgery (T0), and after 6 (T1), 26 (T2), 52 (T3), and 104 (T4) weeks. Differences in 8 parameters between the affected and unaffected arms were calculated. General linear models were computed to examine time, group, and interaction effects.
\end{abstract}

Results. All outcomes changed significantly, mostly nonlinearly, over time (T0-T4). Between $\mathrm{T} 1$ and $\mathrm{T} 4$,

(C) The Author(s) 2010. This article is published with open access at Springerlink.com

First Received: 23 June 2009;

Published Online: 11 March 2010

H. J. Hoekstra, MD, PhD

e-mail: h.j.hoekstra@chir.umcg.nl limitations decreased in abduction (all groups); anteflexion, abduction-exorotation, abduction strength (SLNB + CLND, ALND); flexion strength (SLNB + CLND); and arm volume (SLNB, SLNB + CLND). At T4, limitations in anteflexion (SLNB, ALND), abduction (SLNB + CLND, ALND), exorotation (ALND), abduction-exorotation (all groups), and volume (SLNB + CLND, ALND) increased significantly compared with T0. The SLNB group showed an advantage in anteflexion, abduction, abduction-exorotation, and volume. Groups changed significantly but differently over time in anteflexion, abduction, abduction/ exorotation, abduction strength, flexion strength, and volume. Effect sizes varied from 0.19 to 0.00 .

Conclusion. Initial declines in range of motion and strength were followed by recovery, although not always to presurgery levels. Range of motion and volume outcomes were better for SLNB than ALND, but not strength. SLNB surpassed SLNB + CLND in 2 of the range of motion variables. The clinical relevance of these results is negligible.

Breast cancer is the most common form of cancer in women in the Western world. At some time during their life, breast cancer will be diagnosed in about 1 of every 8 women. ${ }^{1}$ Over the past few years, the 5-year survival rate has increased by about $10 \%$ with the introduction of breast cancer screening and improved adjuvant chemotherapy 
and/or hormonal therapy. ${ }^{2}$ At present, the 5-year survival rate is almost $90 \%{ }^{1}$ Changes in the field of surgical treatment over the past 20 years have involved a decrease in the extent of breast surgery (surgery according to Halstedt, Patey, Madden, and breast-conserving therapy $[\mathrm{BCT}])$ and the introduction of minimally invasive staging of the axilla by means of sentinel lymph node biopsy (SLNB). In women with stage I-II breast cancer whose clinical or ultrasound axillary findings are not suspicious, the introduction of SLNB has meant that, at present, an axillary lymph node dissection (ALND) can be omitted in two-thirds to three-quarters of women. ${ }^{3-5}$

Since the introduction of SLNB in the treatment of breast cancer more than 15 years ago, there have been more than 1600 publications on the clinical application of SLNB in breast cancer (PubMed keywords: sentinel node, breast, study). These studies showed that, despite the less-invasive nature of SLNB, there is still a risk of treatment-related morbidity, such as paraesthesia, limitations in movement of the shoulder and arm, or lymphedema of the arm. ${ }^{5,6}$

A total of 20 studies made objective evaluations of the function of the shoulder/arm and lymphedema of the arm in women with stage I-II breast cancer and compared patients after SLNB with patients after ALND. ${ }^{4,6-24}$ The results of these studies were inconsistent. For example, 12 studies showed fewer functional complaints in the shoulder and arm after SLNB than after ALND. ${ }^{6,9-15,17,18,20,21}$ In contrast, the 8 remaining studies showed similar morbidity complaints after SLNB or ALND. ${ }^{4,7,8,16,22-24}$ The majority of these studies were retrospective and focused on range of motion limitations and the prevalence of lymphedema in the arm. Only 2 retrospective studies and 1 prospective study described objective measurements of muscle strength in the arm. ${ }^{6,9,18}$ Lastly, only 4 studies assessed shoulder/ arm morbidity preoperatively and at multiple time points postoperatively. Three of these studies found less lymphedema after SLNB than after ALND, whereas the fourth study found no difference in lymphedema. ${ }^{8,10,15,20}$ The 2 studies that evaluated range of motion found fewer limitations in 1 functional shoulder measurement after SLNB than after ALND. ${ }^{10,15}$

To gain more insight into the longitudinal course of the function of the shoulder/arm, and the level of lymphedema in the arm in patients with stage I-II breast cancer who had undergone SLNB or SLNB and complete axillary lymph node dissection (CLND), or ALND, we performed a longitudinal study. We expected (1) no preoperative complaints about the range of motion of the shoulder, arm strength, and volume of the arm (lymphedema) on the affected side in any of the women in any of the groups, but expected the presence of these complaints postoperatively; (2) decreases in shoulder/arm complaints in women in the 3 groups over time; and (3) fewer functional shoulder/arm complaints after SLNB than after SLNB + CLND, or ALND.

This study formed part of a large longitudinal prospective study on functional complaints and quality of life after surgical treatment for breast cancer. ${ }^{6,25}$

\section{METHODS}

\section{Patients}

Over a 2-year period, all patients with stage I and II breast cancer at the University Medical Centre Groningen and the Martini Hospital Groningen were informed about this study by the nurse practitioner and invited to participate. Patients with preexisting shoulder complaints who had been treated in the past with surgery, conservative treatment, medication, and/or physiotherapy, and patients with distant metastases were excluded. The study was approved by the Medical Ethics Committees of the 2 hospitals. Participants agreed to take part by signing an informed consent form.

On the day before surgery (T0), the first measurements were taken by a physician or physiotherapist. The measurements were repeated 6 weeks $(\mathrm{T} 1$, median $=$ 1.44 months), 6 months $(\mathrm{T} 2$, median $=5.92$ months $)$, 12 months $(\mathrm{T} 3$, median $=11.99$ months $)$, and 24 months (T4, median $=23.95$ months) postoperatively.

\section{Treatment}

The surgical SLNB technique was described by us previously. ${ }^{26}$ At the 2 hospitals, breast cancer was treated according to the protocol described in the Dutch guidelines for breast cancer. ${ }^{27}$ When metastases were detected in the sentinel lymph node, level I-II CLND was performed within 2 weeks. All patients who received BCT underwent adjuvant radiotherapy to the breast. When indicated, patients received adjuvant systemic and/or hormonal treatment with or without adjuvant locoregional radiotherapy. Postoperatively, all women received written instructions on standardized exercises of the upper extremities to optimize mobility and function of the affected arm. A physiotherapist instructed all patients on how to perform these exercises in a 20-minute session.

\section{Physical Examination}

Range of motion including anteflexion, abduction, exorotation, and combination abduction-exorotation were measured in degrees according to a standardized protocol using a goniometer (Isomed inclinometer, Portland, OR). ${ }^{28}$

Muscle strength in the arm abductors and elbow flexors was measured in Newtons using a handheld dynamometer 
(Citec, Groningen, The Netherlands). ${ }^{29}$ Grip strength was measured in kilograms using a Jamar handheld dynamometer (Sammons Preston Patterson Medical Division, Bolingbrook, IL). ${ }^{30,31}$

Arm measurements were taken at 4-cm intervals using a tape measure, and the volume was calculated in milliliters according to the method described by Sitzia. ${ }^{32}$

Difference scores were used to express functional limitations; the results on the affected side were subtracted from the results on the nonaffected side. The larger the difference in range of motion or strength scores between the left and right shoulder or arm, the greater the functional limitations. The larger the difference in volume between the 2 arms, the more severe the lymphedema in the affected arm. Difference scores were used in the statistical analyses.

\section{Statistical Analyses}

Descriptive statistics (chi-square and $t$ tests) were used to evaluate the data and to test for differences between groups. The general linear model (GLM) procedure was used to calculate longitudinal time, treatment, and interaction (time $\times$ treatment) effects based on the difference scores from the shoulder and arm measurements and arm volume. Posthoc Bonferroni tests were used when the GLM procedure detected group effects. To test whether shoulder/arm function had recovered to the original level 2 years after surgery, the results obtained at T0 (preoperative values) and T4 (2 years after surgery) were compared using paired $t$ tests. To map longitudinal recovery after surgical intervention, paired $t$ tests were also used to compare T1-T4. Effect sizes were calculated to determine the level of clinical relevance of an effect: an effect size between 0.20 and 0.49 reflected a small clinically relevant difference; an effect size between 0.50 and 0.80 indicated a moderate clinically relevant difference, and an effect size $>0.80$ indicated a large clinically relevant difference. ${ }^{33} P$ values $<.05$ were considered significant. All statistical analyses were carried out with SPSS 14 (Statistical Package for Social Sciences, SPSS Inc., Chicago, IL).

\section{RESULTS}

A total of 209 patients were informed about the study before their operation, and invited to participate; of these, 6 patients refused. Postoperatively, 8 patients were found to be not eligible for the study: 3 patients had benign tumors, 2 patients had ductal carcinoma in situ (DCIS), 2 patients had a T4 tumor, and the primary tumor could not be found in 1 patient. Therefore, 195 of the 201 eligible patients participated in this study (97\%). Of these, 61 received SLNB, 59 received SLNB + CLND, and 75 received ALND. T1 measurements were taken from 185 (95\%), T2 measurements from 183 (94\%), T3 measurements from 178 (91\%), and T4 measurements from $171(88 \%)$ patients. The reasons 24 patients provided for dropping out during the study period (10 SLNB, 4 who received SLNB + CLND, and 10 who received ALND) were the following: 7 patients died from the disease, 2 patients developed metastases, 1 patient was excluded due to breast reconstructive surgery, 7 patients stopped because of lack of interest, and 7 patients missed 1 of the measurements (Fig. 1).

The median age of the 171 participants was 54 (range 31-84) years. There were no significant differences in age or body mass index between the 3 groups. As expected, TNM classification, surgical treatment (lumpectomy, radical mastectomy), number of dissected lymph nodes, complications, radiotherapy, and systemic therapy differed significantly between the groups (Table 1). At T0, the 3 groups had comparable difference scores between the affected and nonaffected sides on range of motion, muscle strength, and arm volume (data not shown).

The GLM procedure was performed with the variables in which the three study groups differed, with the exception of tumor stage because this variable was a determinant factor for further adjuvant treatment. This was done to examine the effect of these variables on shoulder and arm functioning and whether including these variables as a between-subjects factor would produce different results from the procedures including groups according to SLNB, SLNB + CLND, or ALND.

\section{Range of Motion}

Significant time effects were found in the difference scores for the 4 functional shoulder measurements (Fig. 2). These changes-over-time were not linear for abduction, anteflexion, and abduction-exorotation. An increase in the limitations in these functions in the affected shoulder at T0-T1 was followed by a decrease in limitations between $\mathrm{T} 1$ and T4 (Fig. 2). The decrease in limitations in abduction in the affected shoulder between $\mathrm{T} 1$ and $\mathrm{T} 4$ was significant in all 3 groups, while the decreases in limitations in anteflexion and abduction-exorotation were significant in the SNLB + CLND and ALND groups. Compared with $\mathrm{T} 0$, at $\mathrm{T} 4$, the decrease in limitations in anteflexion in the affected shoulder at $\mathrm{T} 4$ was significant in the SLNB and ALND groups, decrease in limitations in abduction was significant in the SLNB + CLND and ALND groups, and decrease in limitations in abductionexorotation was significant in all 3 groups. Changes over time in the affected shoulder showed a linear course for exorotation. At T4, the limitations in exorotation in the affected shoulder were significantly larger than at $\mathrm{T} 0$ in the ALND group, but not in the other two groups (Table 2). 
FIG. 1 Flowchart of patients. a Ductal carcinoma in situ. b Occult breast cancer (no primary tumor was found)

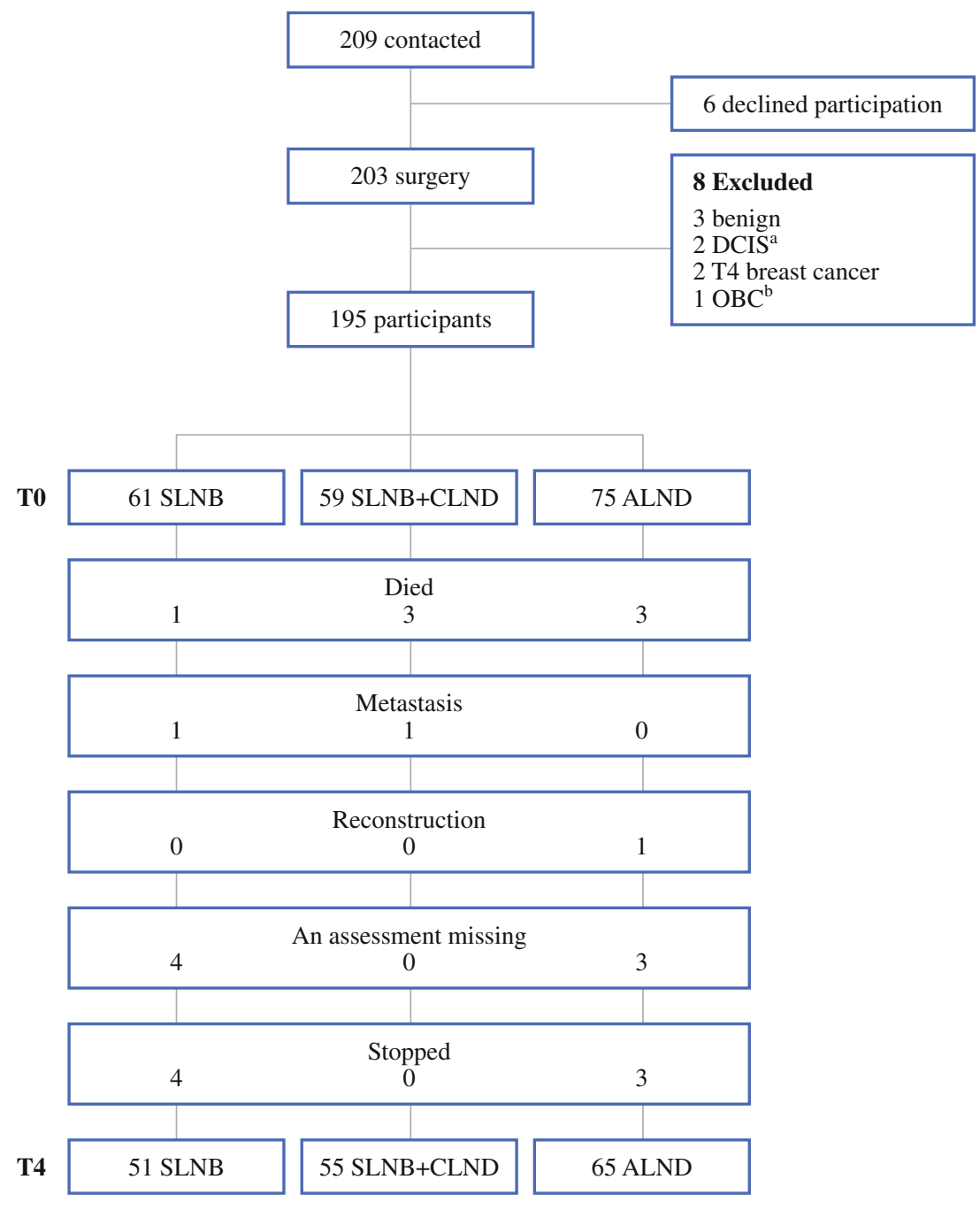

Significant group effects were found in the difference scores for anteflexion, abduction, and abduction-exorotation, but not for exorotation (Fig. 2). These showed an advantage in the SLNB group compared with the SLNB + CLND and ALND groups for anteflexion and abduction, as well as compared with the ALND group for abduction-exorotation (Fig. 2).

Interaction effects (time $\times$ group) were significant for the difference scores in anteflexion, abduction, and abduction-exorotation (Fig. 2). Whereas the limitations in the affected shoulder in the SLNB group remained comparable after $\mathrm{T} 1$ or $\mathrm{T} 2$, limitations in the ALND and SLNB + CLND groups decreased after T1 (Fig. 2). Effect sizes varied between 0.01 and 0.19 (Fig. 2).

\section{Muscle Strength}

Significant time effects were found in the difference scores from the three functional arm measurements
(Fig. 3). The course was not linear. Increases in limitations in the affected arm between T0 and T1 were followed by decreases between T1 and T4 (Fig. 3). These decreases between $\mathrm{T} 1$ and $\mathrm{T} 4$ were significant for abduction strength in the SLNB + CLND and ALND groups, and for flexion strength in the SLNB + CLND group. Changes in limitations in the affected arm in grip strength between $\mathrm{T} 1$ and $\mathrm{T} 4$ were not significant in any of the groups. No significant differences in arm function between $\mathrm{T} 0$ and $\mathrm{T} 4$ were found (Table 2). No significant group effects were found (Fig. 3).

Interaction effects were significant for abduction strength and flexion strength (Fig. 3). Whereas limitations in the affected arm in abduction and flexion strength remained constant in the SLNB group after T1, limitations in abduction strength decreased in the ALND and SLNB + CLND groups, while limitations in flexion strength decreased in the SLNB + CLND group (Table 2). Effect sizes varied between 0.00 and 0.05 (Fig. 3). 
TABLE 1 Patient descriptors and comparisons between groups

\begin{tabular}{|c|c|c|c|c|c|c|c|c|}
\hline \multirow[t]{2}{*}{ Variable } & \multicolumn{2}{|c|}{$\operatorname{SLNB}(N=51)$} & \multicolumn{2}{|c|}{ SLNB + CLND $(N=56)$} & \multicolumn{2}{|c|}{$\operatorname{ALND}(N=65)$} & \multirow[t]{2}{*}{ Test value } & \multirow[t]{2}{*}{$P$} \\
\hline & $N$ & $(\%)$ & $N$ & $(\%)$ & $N$ & $(\%)$ & & \\
\hline Age, mean (SD) & 57.9 & $(11.9)$ & 53.8 & $(9.8)$ & 56.9 & $(11.6)$ & $F=2.30^{\mathrm{b}}$ & .104 \\
\hline TNM classification & & & & & & & $X^{2}=45.11$ & $<.001 *$ \\
\hline Stage I & 38 & $(74.5)$ & 8 & $(14.5)$ & 27 & $(41.5)$ & & \\
\hline Stage II A & 12 & $(23.5)$ & 41 & $(74.5)$ & 26 & $(40.0)$ & & \\
\hline Stage II B & 1 & (2.0) & 6 & (10.9) & 12 & (18.5) & & \\
\hline Surgery & & & & & & & $X^{2}=16.30$ & $<.001$ \\
\hline BCT & 35 & $(68.6)$ & 39 & $(70.9)$ & 25 & $(38.5)$ & & \\
\hline Mastectomy & 16 & $(31.4)$ & 16 & $(29.1)$ & 40 & $(61.5)$ & & \\
\hline Dissected lymph nodes & & & & & & & $X^{2}=158.4$ & $<.001$ \\
\hline$<3$ removed & 48 & & 0 & & 0 & & & \\
\hline $3-10$ removed & 1 & & 20 & & 25 & & & \\
\hline$>10$ removed & 0 & & 32 & & 37 & & & \\
\hline Missing & 2 & & 4 & & 3 & & & \\
\hline Complications & & & & & & & $X^{2}=8.74$ & $.013 * *$ \\
\hline No & 46 & $(89.7)$ & 38 & $(61.5)$ & 56 & $(65.9)$ & & \\
\hline \multicolumn{9}{|l|}{ Yes } \\
\hline$>4$ weeks seroma & 2 & $(4.2)$ & 6 & $(14.0)$ & 11 & $(19.3)$ & & \\
\hline Inflammation $^{\mathrm{a}}$ & 3 & $(6.1)$ & 12 & $(24.5)$ & 8 & $(14.8)$ & & \\
\hline Radiotherapy & & & & & & & $X^{2}=11.47$ & $.003 * * *$ \\
\hline No & 16 & $(31.4)$ & 12 & $(21.8)$ & 33 & $(50.8)$ & & \\
\hline \multicolumn{9}{|l|}{ Yes } \\
\hline Breast & 35 & $(68.6)$ & 39 & $(70.9)$ & 25 & $(38.5)$ & & \\
\hline Breast and axilla & 0 & $(0.0)$ & 4 & (7.3) & 7 & $(10.8)$ & & \\
\hline Systemic therapy & & & & & & & $X^{2}=36.40$ & $<.001 * * * *$ \\
\hline No & 37 & $(72.5)$ & 8 & $(14.5)$ & 28 & $(43.1)$ & & \\
\hline \multicolumn{9}{|l|}{ Yes } \\
\hline Chemo + hormonal - & 4 & (7.8) & 8 & $(14.5)$ & 13 & $(20.0)$ & & \\
\hline Chemo + hormonal + & 5 & (9.8) & 20 & $(36.4)$ & 8 & $(12.3)$ & & \\
\hline Chemo - hormonal + & 5 & (9.8) & 19 & $(34.5)$ & 16 & $(24.6)$ & & \\
\hline Body mass index, mean (SD) & 25.4 & $(4.4)$ & 25.0 & (3.7) & 26.1 & (4.7) & $F=0.26$ & .775 \\
\hline
\end{tabular}

TNM tumor node metastasis, $B C T$ breast-conserving treatment

${ }^{\text {a }}$ Inflammation treated with antibiotics

b One-way ANOVA

* Patients diagnosed with breast cancer stage I versus IIa and IIb

** No complications versus complications

*** No radiotherapy versus radiotherapy

**** No systemic treatment versus systemic treatment

\section{Arm Volume}

Significant time effects, group effects, and interaction effects were found in the arm volume difference scores (Fig. 3). Increases in arm volume were linear in the ALND group, but not in the SLNB or SLNB + CLND groups. At T4, there was a significant increase in arm volume compared with T0 in the SLNB + CLND and
ALND groups, but not in the SLNB group (Table 2, Fig. 3).

Bonferroni tests showed that the increase in volume was significantly smaller in the SLNB group than in the ALND group, but not significantly smaller than in the SLNB + CLND group. The difference between the SLNB + CLND group and the ALND group was not significant. Effect sizes varied between 0.07 and 0.10 (Fig. 3). 


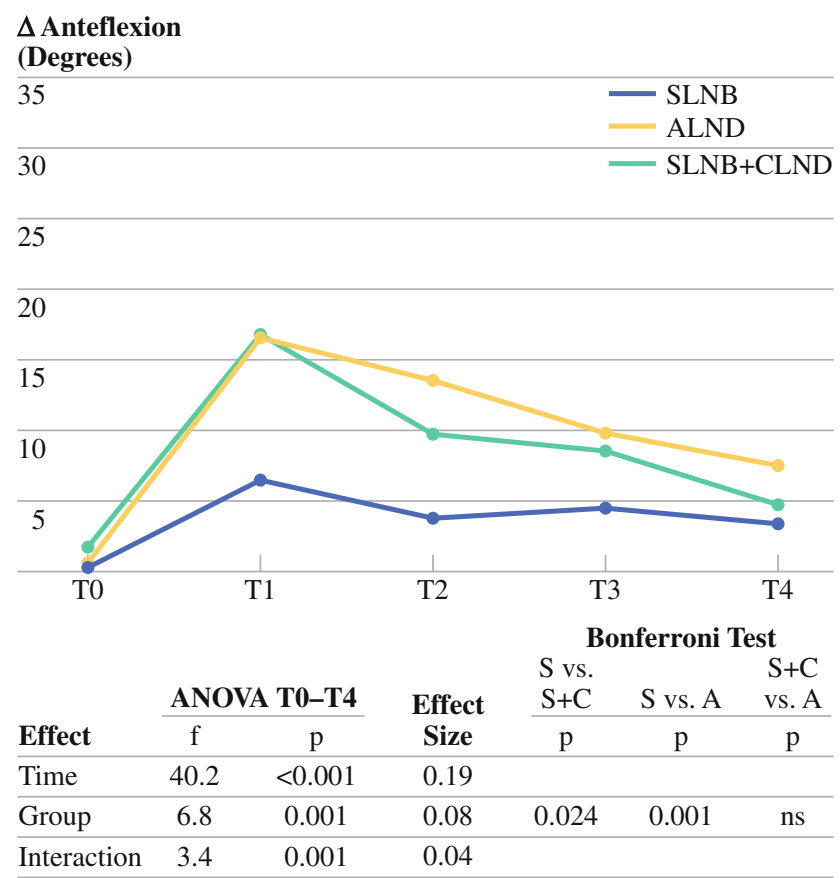

\section{$\Delta$ Exorotation}

(Degrees)

$\begin{aligned} 15 & \\ & \\ & \text { SLNB } \\ & \text { ALND } \\ & \text { SLNB+CLND }\end{aligned}$

10

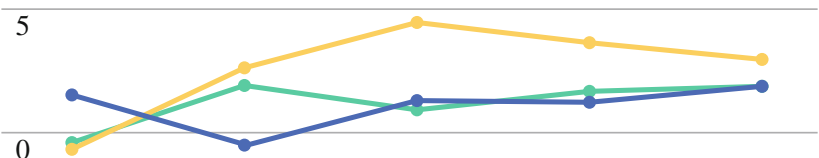

T0

T1

$\mathrm{T} 2$

T3

T4

\begin{tabular}{lccc} 
& \multicolumn{2}{c}{ ANOVA T0-T4 } & Effect \\
\cline { 2 - 3 } Effect & $\mathrm{f}$ & $\mathrm{p}$ & Size \\
Time & 2.6 & 0.031 & 0.02 \\
\hline Group & 0.9 & 0.392 & 0.01 \\
\hline Interaction & 1.5 & 0.135 & 0.02
\end{tabular}

$\Delta$ Abduction

(Degrees)

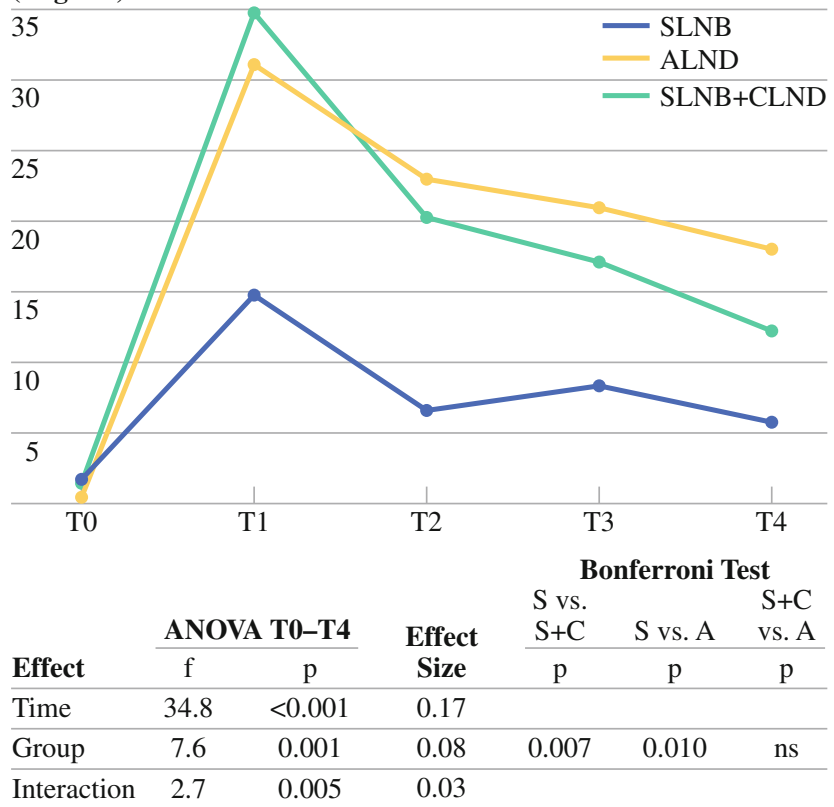

$\Delta$ Abduction-

Exorotation (Degrees)

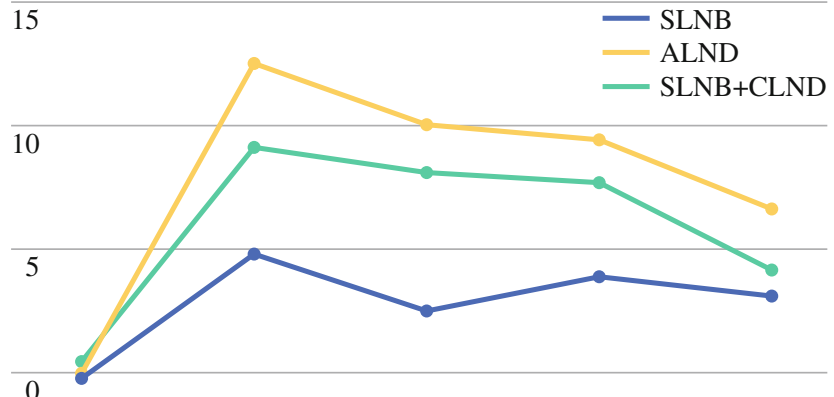

0

T0

T1

T2

T3

T4

Bonferroni Test

\begin{tabular}{lccccccc} 
& \multicolumn{2}{c}{ ANOVA T0-T4 } & Effect & S+C & S vs. A & vs. A \\
Effect & $\mathrm{f}$ & $\mathrm{p}$ & Size & $\mathrm{p}$ & $\mathrm{p}$ & $\mathrm{p}$ \\
\hline Time & 25.3 & $<0.001$ & 0.13 & & & \\
\hline Group & 4.9 & 0.008 & 0.06 & ns & 0.006 & ns \\
\hline Interaction & 2.0 & 0.041 & 0.02 & & &
\end{tabular}

FIG. 2 Results on range of motion. $S$ SLNB, $C$ CLND, A ALND, vs versus

\section{Effect of Treatment-Related Variables}

Type of surgery and complications did not have any significant effect on the difference scores for range of motion, strength, or arm volume. Chemotherapy significantly affected volume only. The consequential Bonferroni tests showed that increases in arm volume were greater in patients who received chemotherapy than in patients who did not receive chemotherapy. Radiation significantly affected anteflexion and abduction. Patients who underwent radiotherapy to the breast and axilla had more limitations in anteflexion and abduction than patients who did not undergo radiotherapy or who received radiotherapy to the breast only. The number of dissected lymph nodes 
TABLE 2 Descriptors of the differences between arms in range of motion, strength, and arm volume, and paired $t$ tests

\begin{tabular}{|c|c|c|c|c|c|c|c|}
\hline Variable & $\begin{array}{l}\text { T0 } \\
\text { Mean (SD) }\end{array}$ & $\begin{array}{l}\text { T1 } \\
\text { Mean (SD) }\end{array}$ & $\begin{array}{l}\text { T2 } \\
\text { Mean (SD) }\end{array}$ & $\begin{array}{l}\text { T3 } \\
\text { Mean (SD) }\end{array}$ & $\begin{array}{l}\text { T4 } \\
\text { Mean (SD) }\end{array}$ & $\begin{array}{l}\mathrm{T} 0-\mathrm{T} 4 \\
P\end{array}$ & $\begin{array}{l}\mathrm{T} 1-\mathrm{T} 4 \\
P\end{array}$ \\
\hline \multicolumn{8}{|l|}{ Range of motion } \\
\hline \multicolumn{8}{|l|}{$\Delta$ ant } \\
\hline SLNB & $0.2(4.1)$ & $6.5(10.6)$ & $3.7(6.1)$ & $4.5(9.1)$ & $3.4(7.8)$ & .008 & 0.079 \\
\hline SLNB + CLND & $1.6(10)$ & $16.6(17)$ & $9.7(12.9)$ & $8.4(14)$ & $4.6(9.4)$ & .053 & $<.001$ \\
\hline ALND & $0.6(5.5)$ & $16.4(16.1)$ & $13.4(19)$ & $9.6(12.4)$ & $7.5(12.8)$ & $<.001$ & $<.001$ \\
\hline \multicolumn{8}{|l|}{$\Delta \mathrm{abd}$} \\
\hline SLNB & 1.7 (12.9) & $14.7(24.2)$ & $6.7(14.7)$ & $8.4(14.6)$ & $5.7(15.2)$ & .194 & .007 \\
\hline SLNB + CLND & $1.4(12.3)$ & $34.7(33.7)$ & $20.3(26.7)$ & $17.1(28.4)$ & $12.2(30)$ & .017 & $<.001$ \\
\hline ALND & $0.4(8.8)$ & $31(29.2)$ & $23(33.5)$ & $20.8(25.6)$ & $18(29.5)$ & $<.001$ & .008 \\
\hline \multicolumn{8}{|l|}{$\Delta$ exo } \\
\hline SLNB & $1.4(6.7)$ & $-0.5(8.3)$ & $1.2(10.2)$ & $1.2(8.6)$ & $1.8(9.6)$ & .757 & .082 \\
\hline SLNB + CLND & $-0.4(7.6)$ & $1.7(8.1)$ & $0.9(8.2)$ & $1.5(9.1)$ & $1.8(8.4)$ & .113 & .816 \\
\hline ALND & $-0.7(7.9)$ & $2.5(9.7)$ & $4.4(15.6)$ & $3.6(10.6)$ & $2.8(8.7)$ & .007 & .837 \\
\hline \multicolumn{8}{|l|}{$\Delta$ abdexo } \\
\hline SLNB & $-0.2(3.1)$ & $4.8(11.5)$ & $2.5(5.1)$ & $3.9(6.7)$ & $3.1(6.1)$ & .001 & .206 \\
\hline SLNB + CLND & $0.38(3.4)$ & $9.1(12.8)$ & $8.1(11.6)$ & $7.7(13.3)$ & $4.1(11.3)$ & .016 & .011 \\
\hline ALND & $-0.1(3.3)$ & $12.4(16.1)$ & $9.9(14.8)$ & $9.4(16.7)$ & $6.7(14.3)$ & $<.001$ & .004 \\
\hline \multicolumn{8}{|l|}{ Strength } \\
\hline \multicolumn{8}{|l|}{$\Delta$ abds } \\
\hline SLNB & $1.5(23.6)$ & $1.2(20.8)$ & $-2.2(26.6)$ & $-7.4(25.1)$ & $3.8(25.6)$ & .454 & .425 \\
\hline SLNB + CLND & $-3.4(26.5)$ & $14.2(34.9)$ & 4.9 (27.7) & $-2.6(31.8)$ & $-2.2(36.5)$ & .813 & .016 \\
\hline ALND & $-5(25.7)$ & $17.8(26.8)$ & 6.7 (28.6) & $6.3(32.4)$ & $0(30.9)$ & .266 & $<.001$ \\
\hline \multicolumn{8}{|l|}{$\Delta$ flexs } \\
\hline SLNB & $2.7(23.1)$ & $0.4(30.7)$ & $5.9(26)$ & $4.1(21.7)$ & $-3.1(25.6)$ & .223 & .653 \\
\hline SLNB+CLND & $-2.2(36.7)$ & $16.9(38.4)$ & $3.5(23.3)$ & $4(28.7)$ & $-0.8(25.8)$ & .928 & .009 \\
\hline ALND & $-3.3(24.2)$ & $11(30.1)$ & $3.5(31.4)$ & $0.6(30.7)$ & $4(27.8)$ & .100 & .081 \\
\hline \multicolumn{8}{|l|}{$\Delta$ grips } \\
\hline SLNB & $0(4.8)$ & $1(5)$ & $0(4.7)$ & $-0.3(4.8)$ & $0.5(3.8)$ & .451 & .365 \\
\hline SLNB+CLND & $0.7(4.5)$ & $1.5(3.7)$ & $1.2(4.5)$ & $1.5(4.5)$ & $0.4(4.2)$ & .656 & .055 \\
\hline ALND & $-0.3(4.4)$ & $1.9(4.9)$ & $1.4(5.1)$ & $1(5.5)$ & $0.8(4.8)$ & .115 & .059 \\
\hline \multicolumn{8}{|l|}{ Arm volume } \\
\hline \multicolumn{8}{|l|}{$\Delta$ vol } \\
\hline SLNB & $14.2(99.5)$ & $0.8(75.4)$ & $4.1(76.2)$ & $-0.1(96.3)$ & $24(77.2)$ & .349 & .015 \\
\hline SLNB + CLND & $-2.3(103.8)$ & $-43.2(97.6)$ & $-46.9(122.1)$ & $-49.5(137.7)$ & $-33.5(148)$ & .041 & .512 \\
\hline ALND & $-4.1(105.7)$ & $-57.3(134)$ & $-76(160.8)$ & $-94.1(189.4)$ & $-116(212)$ & $<.001$ & .010 \\
\hline
\end{tabular}

$\Delta$ unaffected side - affected side, ant anteflexion, abd abduction, exo exorotation, abdexo combined movement of abduction and exorotation, abds abduction strength, flexs flexion strength, grips grip strength, vol arm volume

significantly affected anteflexion, abduction, abduction exorotation, and volume. Patients from whom 3-10 or more than 10 lymph nodes were removed had more limitations in their range of motion than patients who had fewer than 3 nodes removed. Patients who had more than 10 lymph nodes removed had increased arm volume compared with patients who had fewer than 3 or 3-10 nodes removed (Table 3).

\section{DISCUSSION}

In this prospective study in patients with stage I-II breast cancer, validated measurement methods were used to evaluate the function of the shoulder, muscle strength, and volume of the arm over a period of 2 years. It was expected that the limitations in shoulder/arm function would be largest in the immediate postoperative period, 

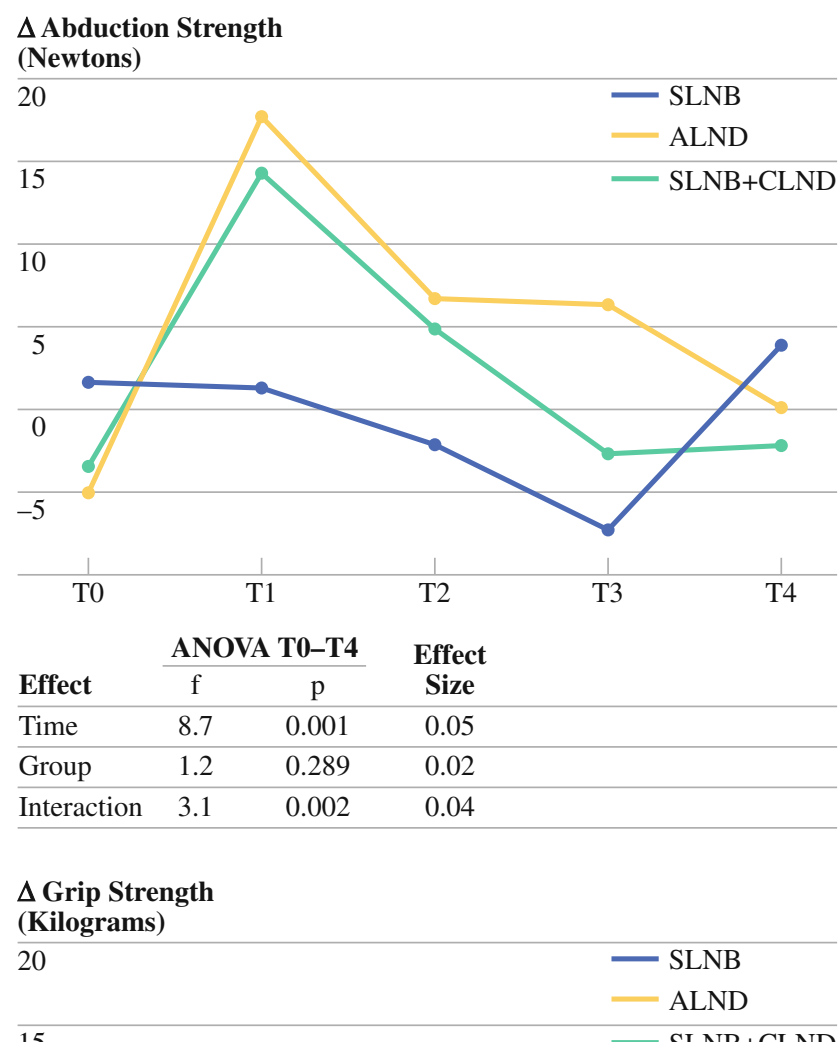

10

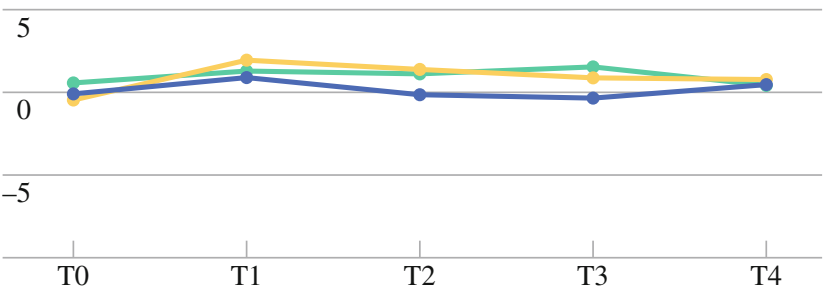

\begin{tabular}{lccc} 
& \multicolumn{2}{c}{ ANOVA T0-T4 } & Effect \\
\cline { 2 - 3 } Effect & $\mathrm{f}$ & $\mathrm{p}$ & Size \\
\hline Time & 3.6 & 0.006 & 0.02 \\
\hline Group & 0.8 & 0.422 & 0.01 \\
Interaction & 1.3 & 0.221 & 0.02
\end{tabular}

$\Delta$ Flexion Strength

(Newtons)
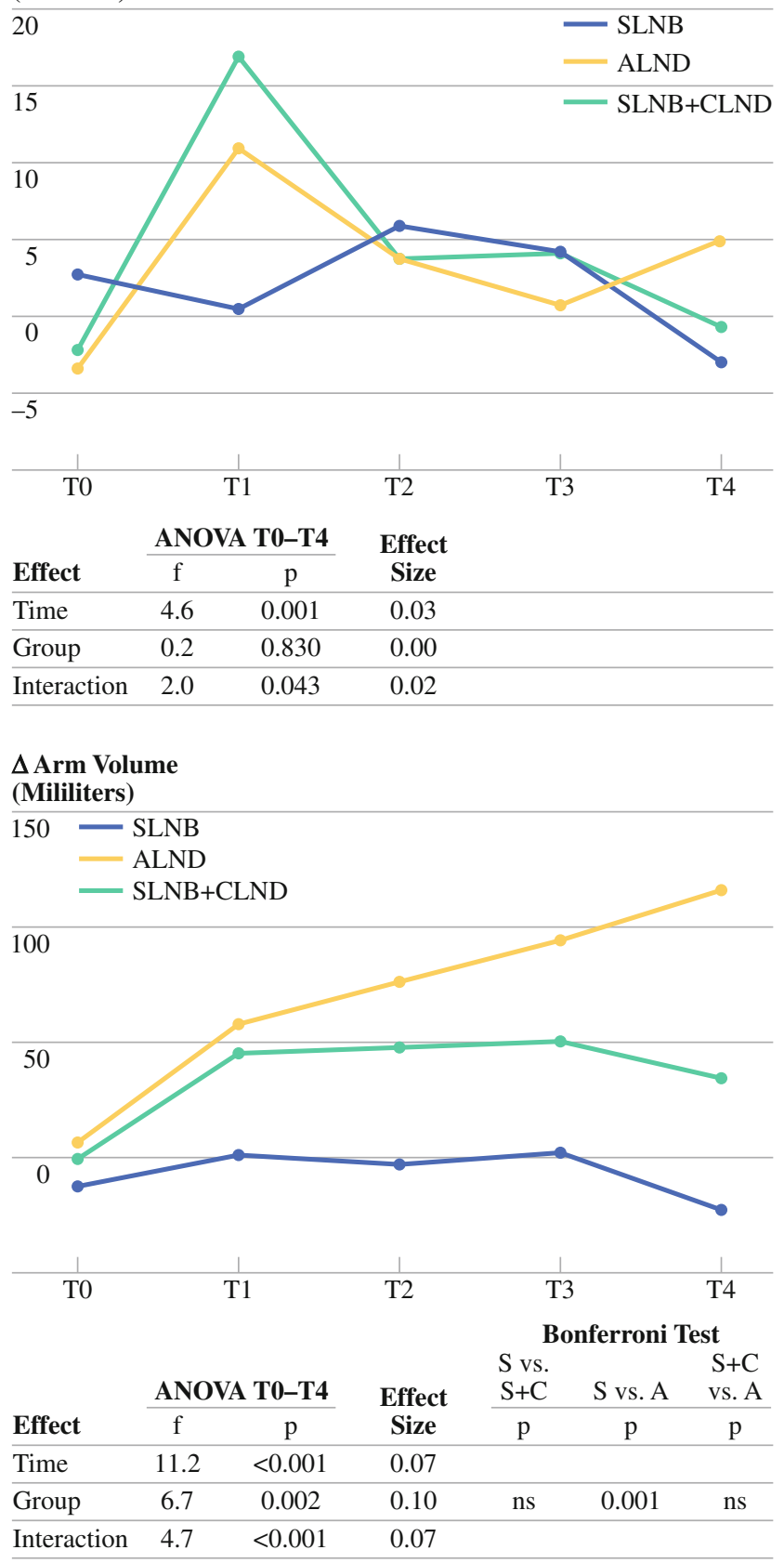

FIG. 3 Results on strength and arm volume. S SLNB, $C$ CLND, A ALND, vs versus

followed by improvement over the course of time, and that the patients in the SLNB group would have less shoulder/ arm morbidity than those in the SLNB + CLND and ALND groups.

\section{Range of Motion}

Our hypothesis that reduced postoperative shoulder function would be followed by improvement over the subsequent 24 months was confirmed in this study.
Recovery of shoulder function was also observed in another study with a shorter follow-up of 12 months. ${ }^{15}$ However, the initial postoperative deterioration in women who underwent ALND was greater than that in the women in the SLNB group. At the 2-year follow-up, women who underwent SLNB still had limitations in 2 of the 4 shoulder functions (anteflexion and abduction-exorotation) compared with the preoperative measurements, whereas women in the ALND group still had limitations in all 4 shoulder functions. Over the 24-month study period, 
TABLE 3 Significant between subjects effects of treatment-related variables, GLM procedure

\begin{tabular}{|c|c|c|c|c|c|c|c|c|c|}
\hline \multirow[t]{2}{*}{ Variable } & \multicolumn{3}{|c|}{ Systemic therapy } & \multicolumn{3}{|c|}{ Radiotherapy } & \multicolumn{3}{|c|}{ Dissected lymph nodes } \\
\hline & $F$ & $P$ & Effect size & $F$ & $P$ & Effect size & $F$ & $P$ & Effect size \\
\hline \multicolumn{10}{|c|}{ Range of motion } \\
\hline$\Delta$ ant & - & ns & - & 3.5 & $.030^{\mathrm{a}}$ & .04 & 6.6 & $.002^{\mathrm{c}}$ & 0.08 \\
\hline$\Delta$ abd & - & $\mathrm{ns}$ & - & 4.6 & $.011^{\mathrm{b}}$ & .05 & 7.3 & $.001^{\mathrm{d}}$ & 0.08 \\
\hline$\Delta$ abdexo & - & ns & - & - & ns & - & 4.4 & $.014^{\mathrm{e}}$ & 0.05 \\
\hline \multicolumn{10}{|c|}{ Arm volume } \\
\hline$\Delta$ vol & 4.9 & .028 & .03 & - & ns & - & 4.8 & $.010^{\mathrm{f}}$ & 0.06 \\
\hline
\end{tabular}

ns not significant

a Bonferroni test: no versus breast ns, no versus breast and axilla $P=.036$, breast versus breast and axilla $P=.031$

b Bonferroni test: no versus breast ns, no versus breast and axilla $P=.018$, breast versus breast and axilla $P=.008$

c Bonferroni test: $<3$ versus 3-10 $P=.003,<3$ versus $>10 P=.012,3-10$ versus $>10 \mathrm{~ns}$

d Bonferroni test: $<3$ versus 3-10 $P=.004,<3$ versus $>10 P=.003,3-10$ versus $>10 \mathrm{~ns}$

e Bonferroni test: $<3$ versus $3-10 P=.042,<3$ versus $>10 P=.024,3-10$ versus $>10$ ns

f Bonferroni test: $<3$ versus $3-10 P=.068,<3$ versus $>10 P=.010,3-10$ versus $>10 \mathrm{~ns}$

women in the SLNB group had fewer limitations in anteflexion, abduction, and abduction-exorotation of the shoulder than women in the SLNB + CLND and ALND groups. This result was in disagreement with 2 other studies that reported initial differences in shoulder function between the treatment groups that were no longer found at the 3-month follow-up. ${ }^{4} 15$ One explanation is that, in these studies, the SLNB group also contained women in whom SLNB had been followed by CLND or radiotherapy to the axilla, owing to inclusion based on the intention to treat principle. Three studies similar to ours and one retrospective study found an advantage in women who underwent SLNB alone, which is in agreement with our results. ${ }^{6,10,11,13}$

\section{Muscle Strength}

This study showed significant changes in muscle strength over time. The patterns differed between the SLNB, SLNB + CLND, and ALND groups. In the axillary lymph node dissection groups (SLNB + CLND and ALND), the postoperative reduction in abduction and flexion strength was larger than that in the SLNB group. All the women (ALND, SLNB + CLND, and SLNB groups) recovered to their preoperative level at the 2-year follow-up. This is in contrast to a 1-year follow-up study that described an advantage of SLNB over ALND. ${ }^{6}$ However, our results also contradict those of 2 retrospective studies that reported an advantage of SLNB over ALND. One of the studies compared women who had undergone SLNB 31 months previously to women who had undergone SLNB + CLND 56 months previously, a difference of 2 years. ${ }^{18}$ In the second study, the results were based on a summation score obtained using a questionnaire that not only measured the subjective experience of muscle strength, but also functional limitations, pain, dysesthesias, and loss of sensitivity. ${ }^{9}$

\section{Arm Volume}

This study confirmed the expectation that arm volume increased less after SLNB than after ALND. It was striking that the volume of the affected arm continued to increase in the ALND group over the course of 2 years, whereas in women who underwent SLNB + CLND, the initial increase was followed by stabilization and, in the SLNB group, there was no change compared with the preoperative arm volume. Over the course of time, arm volume in the ALND group differed significantly from that of the SLNB group, but there was no difference between the SLNB and SLNB + CLND groups. A difference between the SLNB and ALND groups was also found after 1 year in another study. ${ }^{6}$ However, in that study, the last group included ALND and SLNB + CLND patients.

The difference in arm volume between SLNB and ALND and the lack of difference between SLNB and SLNB + CLND agreed with the results of several other studies, of which 2 studies based their results on arm volume and the other studies on changes in 1 or 2 circumferential measurements of the arm. ${ }^{7,8,10,19,22,24}$

In contrast with our results, 2 studies described differences between SLNB and SLNB + CLND. In one of these studies, level III lymph node dissection had been performed. ${ }^{20}$ In the other study, lymphedema was measured objectively and subjectively and the outcomes were combined into 1 variable. Therefore, their "difference" score also contained a subjective component. ${ }^{11}$ 
Our results also contrasted with those of 2 studies that found no difference between SLNB and ALND. ${ }^{4,16}$ One of these studies recruited patients based on the intention to treat principle, so the SLNB group included women in whom SLNB was followed by CLND or radiotherapy to the axilla. ${ }^{4}$ In the other study, group comparisons were made based on differences in the mean increase in arm volume over 6 measurements. ${ }^{16}$ Consequently, the course of the changes in arm volume during follow-up was unclear.

\section{Clinical Relevance}

Although many of the results of our study were statistically significant, the clinical relevance of the time effects and group effects appeared to be negligible for all the variables. However, the standard deviations were wide, which means that there were large differences between the women within each of the groups, and that our population definitely contained women with shoulder/arm functional limitations. It is important to recognize these women at an early stage and, if possible, to refer them for physiotherapy or lymphapress treatment.

\section{Other Treatment-Related Variables}

The type of surgery and complications had no effect on the longitudinal outcomes of shoulder function, arm function, or arm volume. An earlier study reported that women who underwent mastectomy were more likely to report limitations in their range of motion, while the evidence for an effect of complications seems inconclusive. ${ }^{34,35}$ Chemotherapy significantly affected arm volume, which to our knowledge has not been previously reported in the literature. We found that patients who received radiotherapy to the breast and the axilla experienced more range of motion limitations than patients who had not undergone radiotherapy or who had received radiotherapy to the breast only. A previously published study comparing axillary radiation with no axillary radiation in woman after modified radical mastectomy and ALND reported comparable results on range of motion. ${ }^{36}$ Lastly, patients who had more than 3 lymph nodes removed reported having decreased range of motion and increased arm volume compared with patients from whom fewer than 3 nodes were removed. This last finding is not surprising because fewer than 3 lymph nodes were removed in all but 1 of the women in the SLNB group. The results confirm those found for groups according to axillary surgery (SLNB, SLNB + CLND, or ALND).

\section{Study Limitations and Further Comments}

Because of a number of limitations in our study, caution is required in the interpretation of the results and their generalization to the total population of breast cancer patients. The number of participants was small in relation to the number of statistical analyses. All women who participated had stage I-II breast cancer, and only those who were disease-free were included in the longitudinal analyses.

The strengths of our study were the prospective design and that the evaluations were done strictly according to protocol using validated and standardized functional tests. The difference score calculations between the affected and nonaffected sides provided objective insight into the shoulder/arm functional limitations that occurred on a group level. However, the wide standard deviations indicate large differences between individuals in each of the groups.

In conclusion, the limitations in shoulder function and arm strength were most severe immediately after surgery for breast cancer. At the 2-year follow-up, all women had regained their preoperative level of arm muscle strength. However, there were still limitations in their shoulder function. It appeared that women in the SLNB group had fewer limitations. Women in the ALND group showed gradual lymphedema progression during the 2 years of follow-up, whereas the women in the SLNB group did not develop lymphedema. In the SLNB + CLND group, lymphedema occurred immediately after surgery, but did not deteriorate over the 2-year period. The time effects and group effects found in this study were of negligible clinical relevance.

ACKNOWLEDGMENT The study was supported by a grant of the Anna Dorothea Hingst Foundation.

OPEN ACCESS This article is distributed under the terms of the Creative Commons Attribution Noncommercial License which permits any noncommercial use, distribution, and reproduction in any medium, provided the original author(s) and source are credited.

\section{REFERENCES}

1. Jemal A, Siegel R, Ward E, Hao Y, Xu J, Thun MJ. Cancer statistics, 2009. CA Cancer J Clin. 2009;59:225-49.

2. Vervoort MM, Draisma G, Fracheboud J, van de Poll-Franse LV, de Koning HJ. Trends in the usage of adjuvant systemic therapy for breast cancer in the Netherlands and its effect on mortality. $\mathrm{Br}$ J Cancer. 2004;91:242-7.

3. Krag DN, Anderson SJ, Julian TB, Brown AM, Harlow SP, Ashikaga $\mathrm{T}$, et al. Technical outcomes of sentinel-lymph-node resection and conventional axillary-lymph-node dissection in patients with clinically node-negative breast cancer: results from the NSABP B-32 randomised phase III trial. Lancet Oncol. 2007;8:881-8.

4. Mansel RE, Fallowfield L, Kissin M, Goyal A, Newcombe RG, Dixon JM, et al. Randomized multicenter trial of sentinel node biopsy versus standard axillary treatment in operable breast cancer: the ALMANAC Trial. J Natl Cancer Inst. 2006;98:599_ 609. 
5. Wilke LG, McCall LM, Posther KE, Whitworth PW, Reintgen DS, Leitch AM, et al. Surgical complications associated with sentinel lymph node biopsy: results from a prospective international cooperative group trial. Ann Surg Oncol. 2006;13:491-500.

6. Rietman JS, Dijkstra PU, Geertzen JH, Baas P, de Vries J, Dolsma WV, et al. Treatment-related upper limb morbidity 1 year after sentinel lymph node biopsy or axillary lymph node dissection for stage I or II breast cancer. Ann Surg Oncol. 2004; 11:1018-24.

7. Burak WE, Hollenbeck ST, Zervos EE, Hock KL, Kemp LC, Young DC. Sentinel lymph node biopsy results in less postoperative morbidity compared with axillary lymph node dissection for breast cancer. Am J Surg. 2002;183:23-7.

8. Del Bianco P, Zavagno G, Burelli P, Scalco G, Barutta L, Carraro $\mathrm{P}$, et al. Morbidity comparison of sentinel lymph node biopsy versus conventional axillary lymph node dissection for breast cancer patients: Results of the sentinella-GIVOM Italian randomised clinical trial. Eur J Surg Oncol. 2008;34:508-13.

9. Haid A, Kuehn T, Konstantiniuk P, Koberle-Wuhrer R, Knauer M, Kreienberg R, et al. Shoulder-arm morbidity following axillary dissection and sentinel node only biopsy for breast cancer. Eur J Surg Oncol. 2002;28:705-10.

10. Husted MA, Haugaard K, Soerensen J, Bokmand S, Friis E, Holtveg $\mathrm{H}$, et al. Arm morbidity following sentinel lymph node biopsy or axillary lymph node dissection: A study from the Danish Breast Cancer Cooperative Group. Breast. 2008;17:13847.

11. Langer I, Guller U, Berclaz G, Koechli OR, Schaer G, Fehr MK, et al. Morbidity of sentinel lymph node biopsy (SLN) alone versus SLN and completion axillary lymph node dissection after breast cancer surgery: a prospective Swiss multicenter study on 659 patients. Ann Surg. 2007;245:452-61.

12. Leidenius M, Leppanen E, Krogerus L, von Smitten K. Motion restriction and axillary web syndrome after sentinel node biopsy and axillary clearance in breast cancer. Am J Surg. 2003;185: $127-30$.

13. Leidenius M, Leivonen M, Vironen J, von Smitten K. The consequences of long-time arm morbidity in node-negative breast cancer patients with sentinel node biopsy or axillary clearance. $J$ Surg Oncol. 2005;92:23-31.

14. Peintinger F, Reitsamer R, Stranzl H, Ralph G. Comparison of quality of life and arm complaints after axillary lymph node dissection vs sentinel lymph node biopsy in breast cancer patients. Br J Cancer. 2003;89:648-52.

15. Purushotham AD, Upponi S, Klevesath MB, Bobrow L, Millar K, Myles JP, et al. Morbidity after sentinel lymph node biopsy in primary breast cancer: results from a randomized controlled trial. J Clin Oncol. 2005;23:4312-21.

16. Ronka R, von Smitten K, Tasmuth T, Leidenius M. One-year morbidity after sentinel node biopsy and breast surgery. Breast. 2005; 14:28-36.

17. Schrenk P, Rieger R, Shamiyeh A, Wayand W. Morbidity following sentinel lymph node biopsy versus axillary lymph node dissection for patients with breast carcinoma. Cancer. 2000; 88:608-14.

18. Schulze T, Mucke J, Markwardt J, Schlag PM, Bembenek A. Long-term morbidity of patients with early breast cancer after sentinel lymph node biopsy compared to axillary lymph node dissection. J Surg Oncol. 2006;93:109-19.

19. Temple LK, Baron R, Cody HS, III, Fey JV, Thaler HT, Borgen PI, et al. Sensory morbidity after sentinel lymph node biopsy and axillary dissection: a prospective study of 233 women. Ann Surg Oncol. 2002;9:654-62.

20. Veronesi U, Paganelli G, Viale G, Luini A, Zurrida S, Galimberti $\mathrm{V}$, et al. A randomized comparison of sentinel-node biopsy with routine axillary dissection in breast cancer. $N$ Engl J Med. 2003;349:546-53.

21. Crane-Okada R, Wacher RA, Elashoff D, Guiliano AE. Longterm morbidity of sentinel node biopsy versus complete axillary dissection for unilateral breast cancer. Ann Surg Oncol. 2008;15:1996-2005.

22. Zavagno G, De Salvo GL, Scalco G, Bozza F, Barutta L, Del BP, et al. A Randomized clinical trial on sentinel lymph node biopsy versus axillary lymph node dissection in breast cancer: results of the Sentinella/GIVOM trial. Ann Surg. 2008;247:207-13.

23. Arnaud S, Houvenaeghel G, Moutardier V, Butarelli M, Martino $\mathrm{M}$, Tallet A, et al. Patients' and surgeons' perspectives on axillary surgery for breast cancer. Eur J Surg Oncol. 2004;30:735-43.

24. Goyal A, Newcombe RG, Chabra A, Mansel RE. Morbidity in breast cancer patients with sentinel node metastases undergoing delayed axillary lymph node dissection (ALND) compared with immediate ALND. Ann Surg Oncol. 2008;15:262-7.

25. Kootstra J, Hoekstra-Weebers JE, Rietman H, de Vries J, Baas P, Geertzen JH, et al. Quality of life after sentinel lymph node biopsy or axillary lymph node dissection in stage I/II breast cancer patients: a prospective longitudinal study. Ann Surg Oncol. 2008;15:2533-41.

26. Rutgers EJ, Jansen L, Nieweg OE, de Vries J, Schraffordt Koops $\mathrm{H}$, Kroon BB, de Vries JE. Technique of sentinel node biopsy in breast cancer. Eur J Surg Oncol. 1998;24:316-9.

27. Richtlijn mammacarcinoom. Available: www.oncoline.nl/ mamma/mammacarcinoom. Accessed 15 Jan 2010.

28. Green S, Buchbinder R, Forbes A, Bellamy N. A standardized protocol for measurement of range of movement of the shoulder using the Plurimeter- $\mathrm{V}$ inclinometer and assessment of its intrarater and interrater reliability. Arthritis Care Res. 1998; 11:4352.

29. van der Ploeg RJ, Fidler V, Oosterhuis HJ. Hand-held myometry: reference values. J Neurol Neurosurg Psychiatry. 1991;54:244-7.

30. Swedborg I, Borg G, Sarnelid M. Somatic sensation and discomfort in the arm of post-mastectomy patients. Scand J Rehabil Med. 1981;13:23-9.

31. Mathiowetz V, Weber K, Volland G, Kashman N. Reliability and validity of grip and pinch strength evaluations. J Hand Surg (Am). 1984;9:222-6.

32. Sitzia J. Volume measurement in lymphoedema treatment: examination of formulae. Eur J Cancer Care (Engl). 1995;4:116.

33. Cohen J, Statistical Power Analysis for the Behavioural Sciences. Hillsdale, NJ: Lawrence Erlbaum Associates, 1988.

34. Swenson KK, Nissen MJ, Ceronsky C, Swenson L, Lee MW, Tuttle TM. Comparison of side effects between sentinel lymph node and axillary lymph node dissection for breast cancer. Ann Surg Oncol. 2002;9:745-53.

35. Liu CQ, Guo Y, Shi JY, Sheng Y. Late morbidity associated with a tumour-negative sentinel lymph node biopsy in primary breast cancer patients: a systematic review. Eur J Cancer. 2009;45: $1560-8$.

36. Blomqvist L, Stark B, Engler N, Malm M. Evaluation of arm and shoulder mobility and strength after modified radical mastectomy and radiotherapy. Acta Oncol. 2004;43:280-3. 